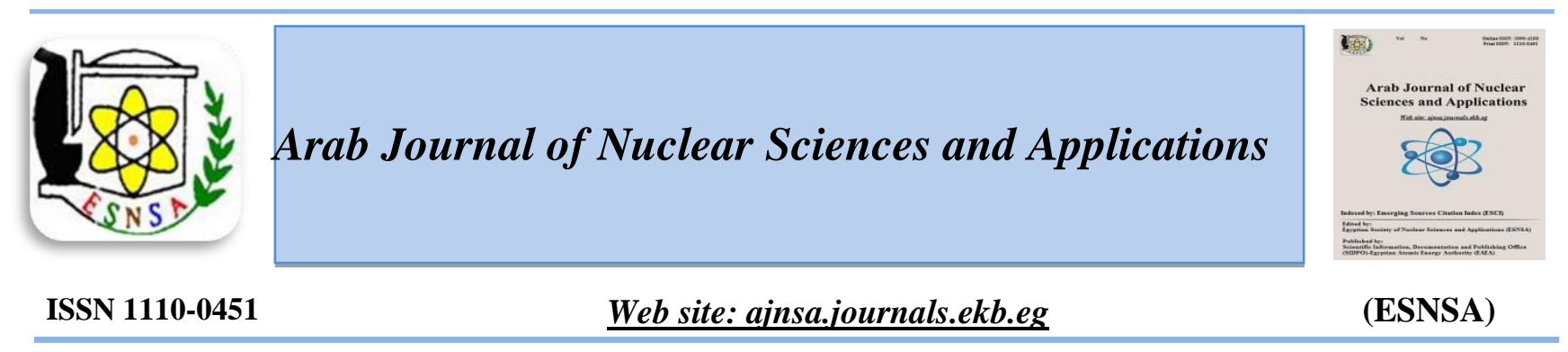

\title{
Deformation and Fracture Behavior of Bicrystal Graphene: an Atomic Level Simulation
}

\author{
A. T. AlMotasem, A. Sedky
}

Physics department- Faculty of Science-Assiut University-71516 Assiut-Egypt

Received $15^{\text {th }}$ Dec. 2018 Accepted $8^{\text {th }}$ May 2019

\begin{abstract}
In this study, extensive molecular dynamics simulations were carried out to investigate failure processes along different symmetric tilt grain boundaries (STGB) of bicrystal graphene sheet. Two different types of STGBs graphene mainly zigzag and arm-chair types were investigated. The dependence of fracture strength, strain as well as Young's moduli on different STGBs were examined. The results clearly show that pristine graphene has the highest values of fracture strength and strain to fracture. Furthermore, bicrystal graphene with zigzag-oriented grain boundaries have improved mechanical properties in comparison to those with arm-chair oriented grain boundaries. Fracture behavior was investigated by applying mode I loadings to the outer boundary of bicrystalline graphene sheet with several misorientation angles. The critical stress intensity factors (SIFs) are calculated as a function of displacement following crack-tip opening displacements (CTOD) method at the incipient bond breaking. The atomistic results show that the crack propagation along armchair-orientation grain boundaries are faster than that of zigzag-orientation grain boundaries of bicrystal graphene.
\end{abstract}

Keywords: Bicrystal/ Grain Boundary/Molecular Dynamics/Deformation/ Fracture.

\section{Introduction}

Graphene is a one-atom-thick layer of carbon atoms with a hexagonal lattice. It attracted great attentions in industrial applications due to its excellent mechanical and electrical properties [13]. For instance, in nuclear industry, graphene is highly considered as candidate material for in-core structural materials for Gen (IV) nuclear energy systems. Also, in High Temperature Gas-cooled Reactors (HTGRs), graphite acts as a good moderator of neutron[4], hence reproducing graphite with high tolerance to irradiation damage which is desirable.

Pristine graphene has extraordinary mechanical properties, arising from high formation energies of defects and strong bonding of carbon atoms[5]. However, large scale production of graphene can be synthesized commercially by using the chemical vapor deposition (CVD) method. Thus, the product is polycrystalline graphene composed of crystallites and grain boundaries (GBs) [6-8]. In polycrystalline graphene, carbon atoms in grain boundaries are less ordered adjacent atoms than those in homogeneous pristine graphene. Such arrangement of atoms in each GBs makes GBs favorit path for crack propagation which eventually lead to loss of their mechanical properties.

Experimental studies on the mechanical properties of graphene reported that it is one of the most strong materials having an elastic modulus of 1.0 $\mathrm{TPa}[9,10]$, which exceeds those of any previously existing materials for instance steels. In their recent experiment, Zhang et al [11] have shown experimentally that the fracture toughness of graphene, measured as the critical stress intensity factor, is about $4.0 \pm 0.6\left(\mathrm{MPa} \cdot \mathrm{m}^{1 / 2}\right)$ which corresponds to a critical strain energy release rate of $15.9 \mathrm{~J} \mathrm{~m}^{-2}$. On the other hand, Cao et al [12] found that the strength of bicrystal graphene varies with the mis-orientation angle. For instance, it was

Corresponding author: a.almotasem@aun.edu.eg

DOI: 10.21608/ajnsa.2019.6677.1155

(C) Scientific Information, Documentation and Publishing Office (SIDPO)-EAEA 
reported that the strength of zigzag-oriented graphene is slightly larger than that of armchairoriented graphene. Such behavior could be attributed to difference of structure and periodicity of each grain boundary. These differences lead to creation of heterogeneous stress field around the crack tip.

Mortazavi and Cuniberti [13] have studied the strength of polycrystalline graphene by means of molecular dynamics simulations. They compared the strength of pristine graphene with poly crystalline graphene with different grain sizes. Their results showed that the mechanical properties of graphene are lower when grain size decreases. However, they discovered that continuous decrease of grain size up to ultrafine grain leads to strength that is similar to that of pristine graphene.

In the present study, deformation and fracture of graphene bicrystal along symmetric tilt grain boundaries (STGBs) are investigated by means of molecular statics/dynamics simulation, in which the adaptive intermolecular reactive empirical bond order (AIREBO) potential [14] is employed. The critical stress intensity factors (SIFs), as a function of the mode I loading, were evaluated at the onset of bond breaking for different (STGBs). These investigations are crucial for further development on using graphene in Nanoelectromechanical systems (NEMS) and Microelectromechanical systems (MEMS).

\section{Simulation Methodology \\ Construction of GB Model}

To model graphene bicrystal containing different sigma tilt grain boundaries (STGBs), a rectangular sheet of graphene is cut along the line passing through the reference point and the point represents the target (STGB). The tilt angle ( $\theta_{\mathrm{TA}} / 2$ ), defined as half sigma tilt angle, represents the angle between the line which the cut is made and the reference line, thus $\Sigma 1$ corresponds to $30^{\circ}$. The newly two created sheets are then rotated and joined along the symmetry line as shown in Figure 1.

Five different STGBs are considered in this work, $\Sigma 1, \Sigma 93, \Sigma 79, \Sigma 39, \Sigma 31 . \quad \Sigma 1$ represents pristine graphene, while ${ }^{\Sigma 31, \Sigma 39}$ represent armchairoriented grain boundary and $\Sigma 93, \Sigma 79$ mark zigzag-oriented grain boundary. A rectangular simulation cells, about ( $380 \AA \times 250 \AA$ ), containing approximately 43000 carbon atoms were used. The interaction between carbons atoms are described by employing the newly constructed adaptive intermolecular reactive bond order (AIREBO) potential [14]. The AIREBO potential correctly reproduces the mechanical properties of graphene sheet including formation and breaking of bonds[16-18].

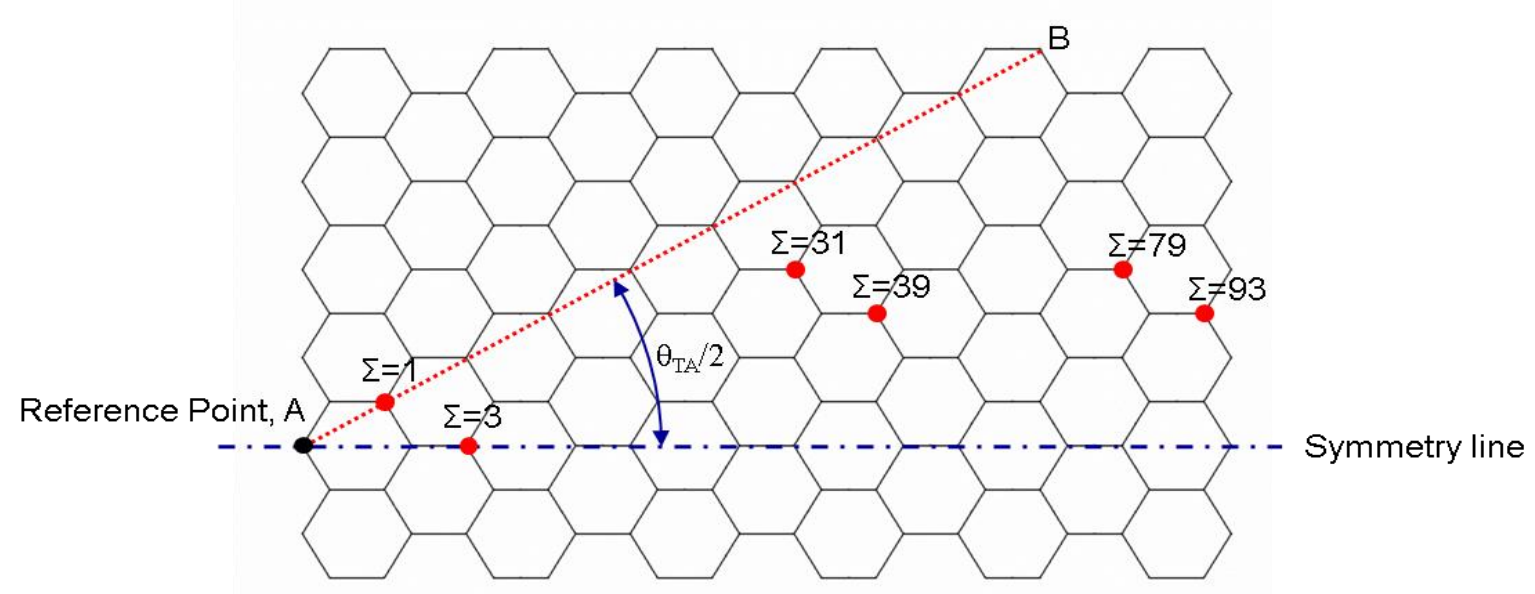

Fig. (1): Schematic diagram illustrates different STGB of monolayer graphene adapted from Ref [15] 
Table (1): Different parameters of STGBs bicrystalline graphene

\begin{tabular}{ccc}
\hline $\begin{array}{c}\text { Sigma } \\
\text { number }\end{array}$ & $\begin{array}{c}\theta_{\text {TA }} \\
\text { degree }\end{array}$ & $\begin{array}{c}\text { Periodic } \\
\text { Length }(\AA)\end{array}$ \\
\hline$\Sigma 1$ & $0.00^{\circ}$ & 2.46 \\
$\Sigma 93$ & $17.90^{\circ}$ & 23.66 \\
$\Sigma 79$ & $26.01^{\circ}$ & 21.83 \\
$\Sigma 39$ & $27.79^{\circ}$ & 15.31 \\
$\Sigma 31$ & $42.10^{\circ}$ & 13.63 \\
\hline
\end{tabular}

MD Simulations are performed using the opensource Larger-scale Atomic/Molecular Massively Parallel Simulator (LAMMPS)[19]. Periodic boundary conditions are imposed on $\mathrm{x}, \mathrm{y}$ and $\mathrm{z}$ directions. Initially, the generated configuration is equilibrated using conjugate gradient algorithm. After that, the system equilibrated for 20 ps using isobaric-isothermal (NPT) ensemble, and then the bicrystal uniaxially deformed along y-direction with strain rate $5 \times 10^{-8} \mathrm{~s}^{-1}$ with a time step of 0.5 fs with NVE microcanonical ensemble. All visualization snapshots are made via visual molecular dynamics (VMD) [20].

\section{Calculation of stress intensity factors}

In order to investigate the fracture toughness of the bicrystal graphene we resort to the linear elastic fracture mechanics (LEFM) model. In this model, the displacement fields, $\mathrm{u}_{\mathrm{x}}$ and $\mathrm{u}_{\mathrm{y}}$, around an edge crack tip is given by

$$
\begin{aligned}
& \mathrm{u}_{\mathrm{x}}=\frac{\mathrm{K}_{\mathrm{I}}}{\mu} \sqrt{\frac{\mathrm{r}}{2 \pi}} \cos \left(\frac{\theta}{2}\right)\left[\frac{1}{2}(\kappa-1)+\sin ^{2}\left(\frac{\theta}{2}\right)\right] \\
& +\frac{\mathrm{K}_{\mathrm{II}}}{\mu} \sqrt{\frac{\mathrm{r}}{2 \pi}} \sin \left(\frac{\theta}{2}\right)\left[\frac{1}{2}(\kappa+1)+\cos ^{2}\left(\frac{\theta}{2}\right)\right] \\
& \mathrm{u}_{\mathrm{y}}=\frac{\mathrm{K}_{\mathrm{I}}}{\mu} \sqrt{\frac{\mathrm{r}}{2 \pi}} \sin \left(\frac{\theta}{2}\right)\left[\frac{1}{2}(\kappa+1)-\cos ^{2}\left(\frac{\theta}{2}\right)\right] \\
& +\frac{\mathrm{K}_{\mathrm{II}}}{\mu} \sqrt{\frac{\mathrm{r}}{2 \pi}} \cos \left(\frac{\theta}{2}\right)\left[\frac{1}{2}(1-\kappa)+\sin ^{2}\left(\frac{\theta}{2}\right)\right]
\end{aligned}
$$

Where $\kappa=(3-v) /(1+v)$, and $v$ and $\mu$ are the Poisson ratio and shear modulus, respectively. The values of $\nu=0.22$ and $\mu=280 \mathrm{GPa}$ are used for monolayer graphene have been taken from Ref. [21]. $\mathrm{K}_{\mathrm{I}}$ and $\mathrm{K}_{\mathrm{II}}$ are defined as the stress intensity factors of mode I and mode II, respectively. To create an edge crack along the STGB of bicrystal graphene, the displacement fields $u_{x}$ and $u_{y}$ given by Eqs. (1) and (2) with $\mathrm{K}_{\mathrm{I}}^{0}=0.6 \mu \sqrt{\mathrm{a}_{0}}$, while the value of $K_{\|}^{0}=0$, are initially applied to the interior atoms (green atoms) as shown in Figure 2. Note that the boundary atoms (blue atoms) are kept fixed during simulations.

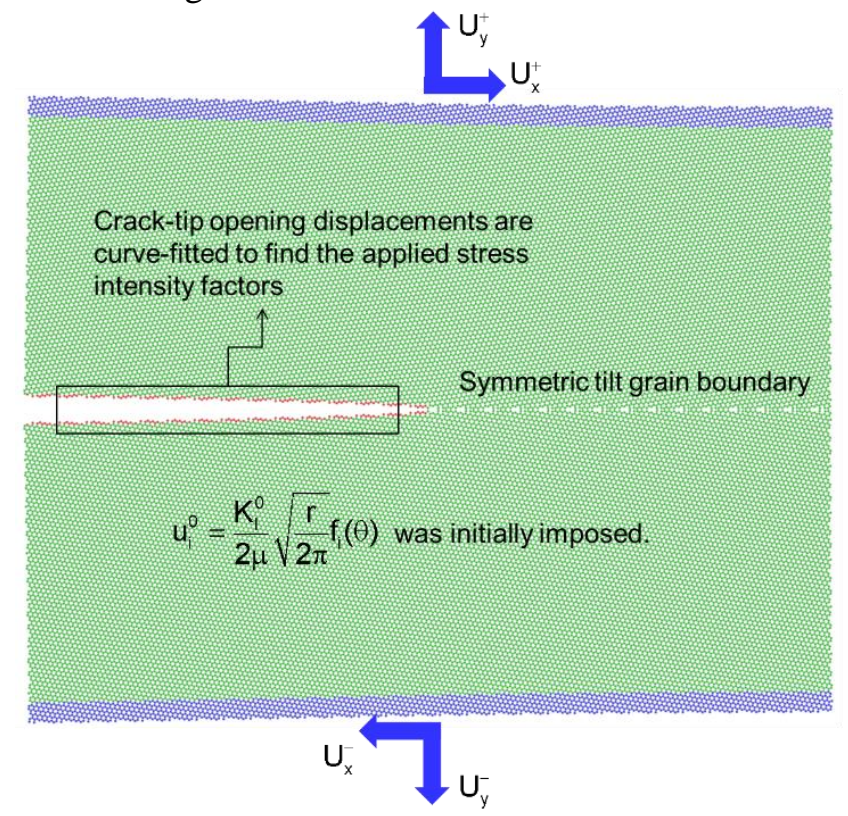

Fig. (2): Initial configuration of $\Sigma 31$ symmetric tilt grain boundary graphene

The minimum energy configurations of the generated edge crack and surrounding carbon atoms, green and red atoms, within the STGB bicrystal graphene can be obtained by applying conjugate gradient method implemented within LAMMPS [19]. The interaction between carbon atoms was determined in which AIREBO potential [22] was used to describe the C-C interactions. Then, a controlled displacement $\mathrm{U}_{\mathrm{y}}^{+}=0.1 \AA$ and $\mathrm{U}_{\mathrm{y}}^{-}=-0.1 \AA$ was applied on the upper and lower boundary atoms (purple atoms). Again, the energy minimization is performed to relax the system. In order to allow for the crack tip to propagate, the last two steps are repeated, and the coordinates of crack tip position is recorded. 


\section{Results And Discussion}

\section{Tensile Deformation}

Figure 3 displays the typical stress-strain curves of bicrystal graphene with different STGBs. From the slopes, in elastic regime, the Young's moduli are easily calculated. Additionally, the values of ultimate tensile strength (UTS) and the fracture strain are determined and listed in Table .2. It is clear that, pristine graphene has the largest value of Yong's modulus (about 850.2 GPa) which is consistent with the reported data for theoretical [23] and experimental [9, 10] studies. Also, it is found that pristine graphene has the highest ultimate tensile strength and fracture strain compared to bicrystal graphene. In the case of STGBs, the values of UTS and fracture strain of armchair-oriented GBs are significantly greater than those of zigzag-oriented GBs. Besides, we find that the value of UTS increases as the tilt angle increases for both armchair and zigzagoriented GBs ranging between 20 30 GPa. Similarly, the value of the fracture strains varies is increasing with tilt angle from $19-34 \%$. In comparison with experiments, the atomistic results show the lower limit of the above data agrees very well with the experimental value of threshold strain beyond which mechanical failure often occurred in polycrystalline graphene.

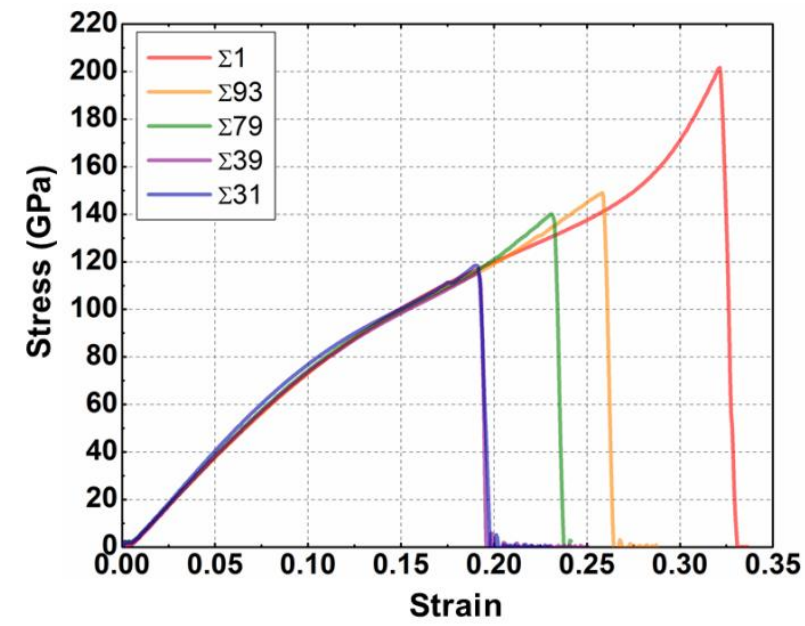

Fig. (3): Typical stress-strain curves of bicrystal graphene with different STGB

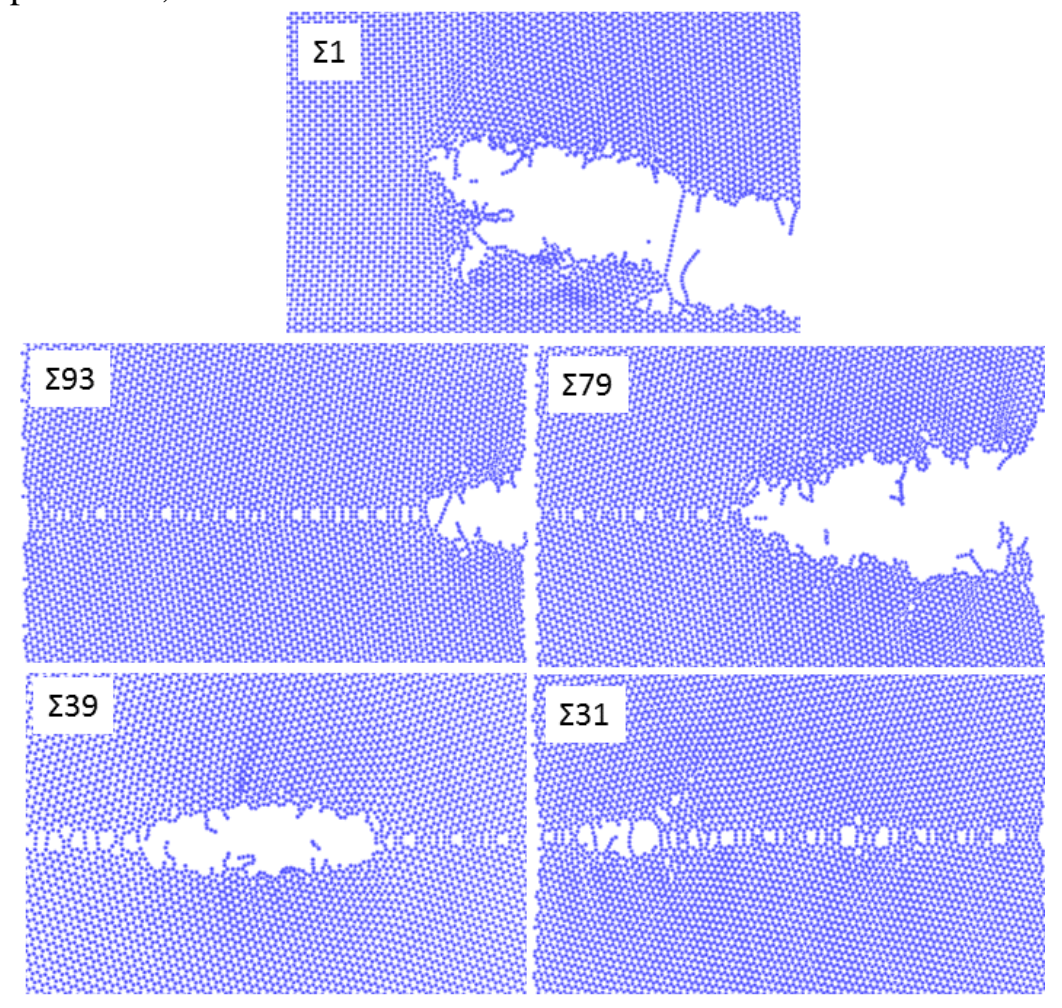

Fig. (4): MD snapshots showing deformation behavior of bicrystalline graphene with different STGB 
Table (2): Mechanical properties, Young's modulus (Y), ultimate tensile strength (UTS) and fracture strain for different STGBs of bicrystal graphene

\begin{tabular}{llll}
\hline $\begin{array}{l}\text { Sigma } \\
\text { number }\end{array}$ & $\begin{array}{l}\text { Fracture } \\
\text { Strain }\end{array}$ & $\begin{array}{l}\text { UTS } \\
(\mathrm{GPa})\end{array}$ & $\begin{array}{l}\text { Y } \\
(\mathrm{GPa})\end{array}$ \\
\hline$\Sigma 1$ & 0.3214 & 201.58 & 850.2 \\
$\Sigma 93$ & 0.2582 & 148.92 & 840.6 \\
$\Sigma 79$ & 0.2308 & 140.03 & 835.4 \\
$\Sigma 39$ & 0.1911 & 115.29 & 825.8 \\
$\Sigma 31$ & 0.19 & 118.4 & 818.1 \\
\hline
\end{tabular}

It was noticed that the failure of the bi-crystal graphene exhibits two different failures. For $\Sigma 93$ and $\Sigma 79$, failure occurs by formation and propagation of crack through the STGB. While in the case of $\Sigma 39$ and $\Sigma 31$, failure is characterized by formation and growth of elliptical cracks. In general, the incipient failure is denoted by the formation of strings known as monoatomic carbon chains (MACCs). The MACCs are gradually stretched up to a few nanometers as the boundary displacement increases. This finding is clearly in agreement with the experimentally observed MACCs strings during elongation of graphene [24].

Stress intensity factors and fracture toughness

Figure 5 displays atomistic visualization of the propagation of edge crack along the two different STGBs of bicrystal graphene, namely $\Sigma 1$ and $\Sigma 1$. $\Sigma 1$ ?repeated?? of STGB graphene is pristine graphene, i.e. has no grain boundary. Therefore, the crack along $\Sigma 1$ STGB exhibits kink into armchair direction, i.e. $30^{\circ}$ inclined from the
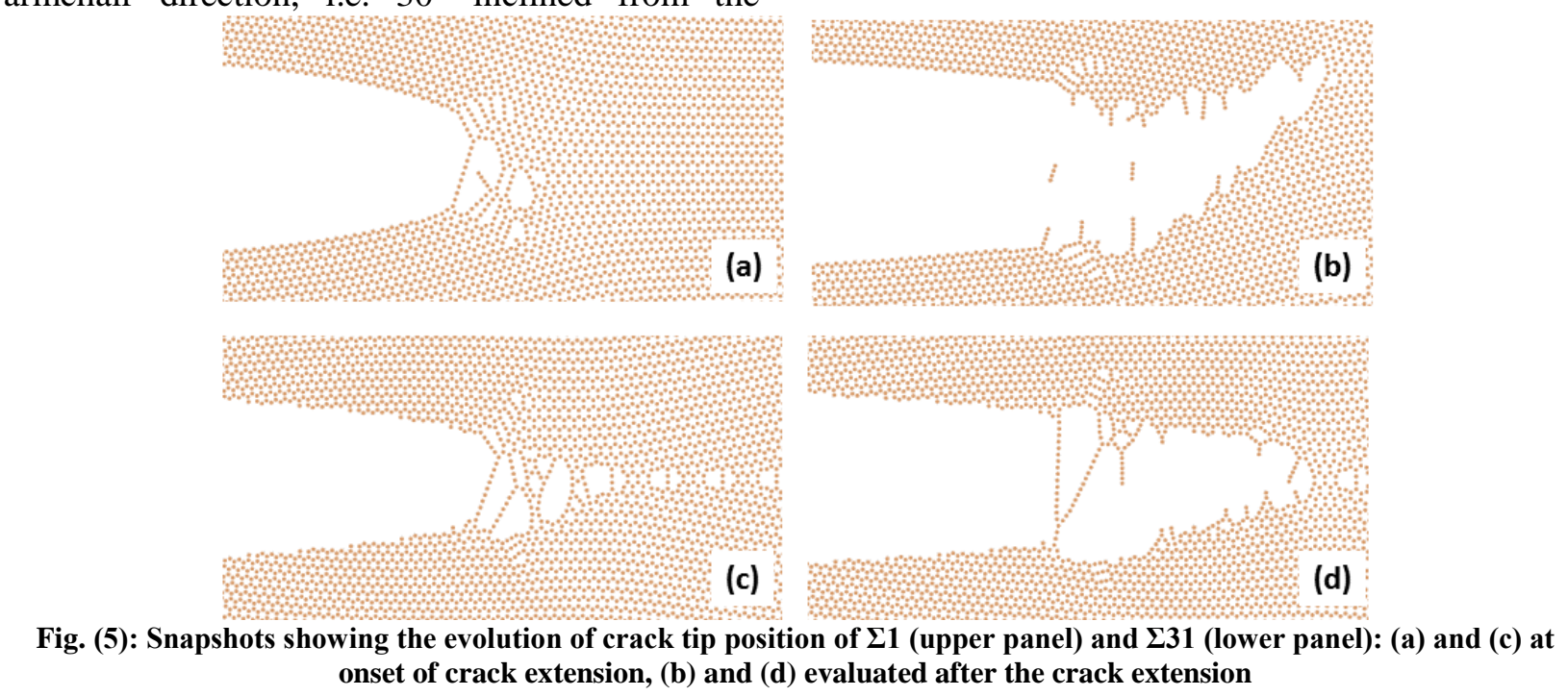

Fig. (5): Snapshots showing the evolution of crack tip position of $\Sigma 1$ (upper panel) and $\Sigma 31$ (lower panel): (a) and (c) at onset of crack extension, (b) and (d) evaluated after the crack extension zigzag direction) as shown in Figs. 5 (a) and (b). It can be seen that armchair-like directions are favoured for crack extension in in comparison to that of zigzag-like STGBs. On the other hand, it was observed that all other interfacial cracks along STGBs of graphene tend to proceed along the grain boundaries as shown in Figure 5 (c) and (d). In order to quantify the fracture toughness, the stress intensity factors (SIFs) are evaluated by fitting the curve of crack-tip opening displacements (CTOD), $\Delta \mathrm{u}_{\mathrm{y}}=\mathrm{u}_{\mathrm{y}}^{+}-\mathrm{u}_{\mathrm{y}}^{-} \quad$ and $\Delta \mathrm{u}_{\mathrm{x}}=\mathrm{u}_{\mathrm{x}}^{+}-\mathrm{u}_{\mathrm{x}}^{-}$, with the formulae, $\Delta u_{y}=b_{1} \sqrt{x-c} \quad$ and $\quad \Delta u_{x}=b_{2} \sqrt{x-c}$, respectively. Here, $b_{1}$ and $b_{2}$ represent fitting parameters corresponding related to $K_{\mathrm{l}}$ and $K_{\mathrm{II}}$ modes, respectively, and $\mathrm{C}$ denotes the crack tip position. The evolution of $K_{\mathrm{I}}$ as a function of the relative displacement, $\Delta \mathrm{U}_{\mathrm{y}}$ is displayed in Figure 6. Generally, the value of $K_{\mathrm{l}_{\text {increases }}}$ with increasing $\Delta \mathrm{u}_{\mathrm{y}}$ up to a maximum then decreases. The critical value of SIFs ( $\left.K_{l}^{\mathrm{c}}\right)$ corresponds to the maximum values of SIFs, i.e. at the onset of crack propagation along the STGBs. It is obvious that the highest value of critical SIFs of mode I is observed for $\Sigma 1$ compared to others of STGBs bicrystals graphene. 
Furthermore, we noticed that the values of $K_{1}^{\mathrm{c}}$ for $\Sigma 93$ and $\Sigma 79$ are comparably higher than those of $\Sigma 39$ and $\Sigma 31$ suggesting that the zigzag-oriented GBs exhibit stronger resistance to crack propagation along STGB in comparison to armchair-oriented GBs.

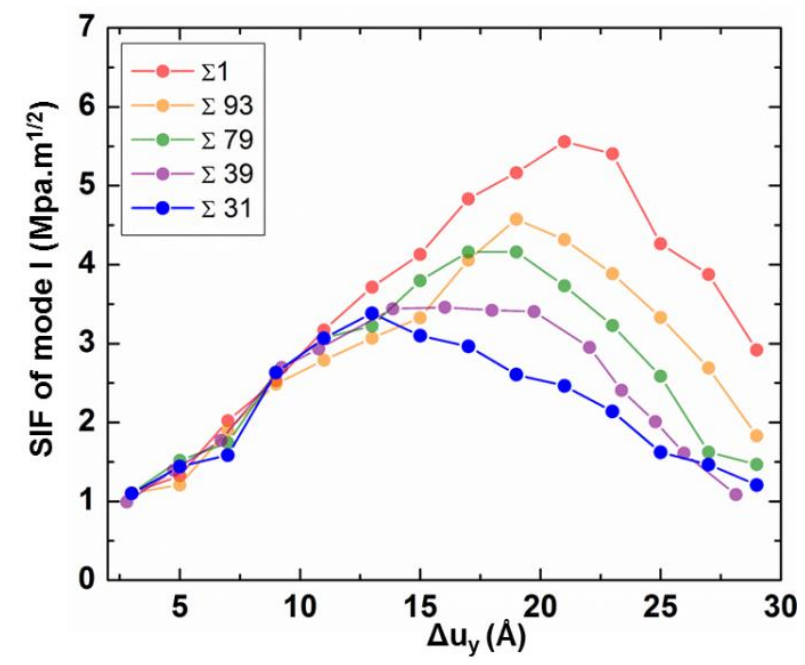

Fig. (6): Variation of $K_{\mathrm{l}}$ vs. ${ }^{\Delta \mathrm{U}_{\mathrm{y}}}$ for different bicrystals graphene

\section{Conclusion}

Deformation and fracture processes along the STGBs of bicrystal graphene are analyzed via MD simulation. The atomistic results show that introducing GB leads to lowering mechanical properties of graphene. For instance, Young's modulus, ultimate tensile strength as well as strain to fracture are reduced by inclusion of STGBs. On the other hand, fracture toughness was examined for different STGB graphene bicrystal with a preexisting an edge crack. The critical SIFs are estimated using fitting the vertical displacement of outer boundary to linear elastic fracture mechanics and crack tip opening displacements method at the incipient of bond breaking. The results indicate that, pristine graphene has the highest value of critical SIFs as illustrated by $\Sigma 1$ compared to graphene with STGBs. We concluded that the crack propagation along STGBs of graphene is significantly influenced by nature of structure and periodicity of GBs. Based on finding of this study, zigzag-oriented GBs exhibit relatively improved mechanical properties than those of armchairoriented GBs in bicrystal graphene.

\section{Acknowledgement}

We are grateful to the High Availability Super Computer Center (HASCC) at Assiut University for providing computational resources.

\section{References}

1-Cao, A., Qu, J.: Atomistic simulation study of brittle failure in nanocrystalline graphene under uniaxial tension. Appl. Phys. Lett. 102, 071902 (2013). doi:10.1063/1.4793088.

2-Balandin, A.A., Ghosh, S., Bao, W., Calizo, I., Teweldebrhan, D., Miao, F., Lau, C.N.: Superior Thermal Conductivity of Single-Layer Graphene. Nano Lett. 8, 902-907 (2008). doi:10.1021/n10731872.

3-Novoselov, K.S., Geim, A.K., Morozov, S.V., Jiang, D., Zhang, Y., Dubonos, S.V., Grigorieva, I.V., Firsov, A.A.: Electric Field Effect in Atomically Thin Carbon Films. Science. 306, 666-669 (2004). doi:10.1126/science.1102896.

4-Allen, T., Busby, J., Meyer, M., Petti, D.: Materials challenges for nuclear systems. Mater. Today. 13, 14-23 (2010). doi:10.1016/S13697021(10)70220-0.

5-Banhart, F., Kotakoski, J., Krasheninnikov, A.V.: Structural Defects in Graphene. ACS Nano. 5, 26-41 (2011). doi:10.1021/nn102598m.

6-Kim, D.W., Kim, Y.H., Jeong, H.S., Jung, H.-T.: Direct visualization of large-area graphene domains and boundaries by optical birefringency. Nat. Nanotechnol. 7, 29-34 (2012). doi:10.1038/nnano.2011.198.

7-Lahiri, J., Lin, Y., Bozkurt, P., Oleynik, I.I., Batzill, M.: An extended defect in graphene as a metallic wire. Nat. Nanotechnol. 5, 326-329 (2010). doi:10.1038/nnano.2010.53.

8-Yu, Q., Jauregui, L.A., Wu, W., Colby, R., Tian, J., Su, Z., Cao, H., Liu, Z., Pandey, D., Wei, D., Chung, T.F., Peng, P., Guisinger, N.P., Stach, E.A., Bao, J., Pei, S.-S., Chen, Y.P.: Control and characterization of individual grains and grain boundaries in graphene grown by chemical vapour deposition. Nat. Mater. 10, 443-449 (2011). doi:10.1038/nmat3010.

9-Lee, C., Wei, X., Kysar, J.W., Hone, J.: Measurement of the Elastic Properties and Intrinsic Strength of Monolayer Graphene. Science. 321, 385-388 (2008). doi:10.1126/science.1157996. 
10-Cao, Q., Geng, X., Wang, H., Wang, P., Liu, A., Lan, Y., Peng, Q.: A Review of Current Development of Graphene Mechanics. Crystals. 8, 357 (2018). doi:10.3390/cryst8090357.

11-Zhang, P., Ma, L., Fan, F., Zeng, Z., Peng, C., Loya, P.E., Liu, Z., Gong, Y., Zhang, J., Zhang, X., Ajayan, P.M., Zhu, T., Lou, J.: Fracture toughness of graphene. Nat. Commun. 5, (2014). doi:10.1038/ncomms4782.

12-Cao, A., Yuan, Y.: Atomistic study on the strength of symmetric tilt grain boundaries in graphene. Appl. Phys. Lett. 100, 211912 (2012). doi:10.1063/1.4722786.

13-Mortazavi, B., Cuniberti, G.: Atomistic modeling of mechanical properties of polycrystalline graphene. Nanotechnology. 25, $215704 \quad$ (2014). doi:10.1088/0957$4484 / 25 / 21 / 215704$.

14-Stuart, S.J., Tutein, A.B., Harrison, J.A.: A reactive potential for hydrocarbons with intermolecular interactions. J. Chem. Phys. 112, 6472-6486 (2000). doi:10.1063/1.481208.

15-Carlsson, J.M., Ghiringhelli, L.M., Fasolino, A.: Theory and hierarchical calculations of the structure and energetics of [0001] tilt grain boundaries in graphene. Phys. Rev. B. 84, (2011). doi:10.1103/PhysRevB.84.165423.

16-Wang, S., Yang, B., Zhang, S., Yuan, J., Si, Y., Chen, H.: Mechanical Properties and Failure Mechanisms of Graphene under a Central Load. ChemPhysChem. 15, 2749-2755 (2014). doi:10.1002/cphc.201402258.

17-Zhao, H., Min, K., Aluru, N.R.: Size and Chirality Dependent Elastic Properties of
Graphene Nanoribbons under Uniaxial Tension.

Nano Lett. 9, 3012-3015 (2009). doi:10.1021/n1901448z.

18-Grantab, R., Shenoy, V.B., Ruoff, R.S.: Anomalous Strength Characteristics of Tilt Grain Boundaries in Graphene. Science. 330, 946-948 (2010). doi:10.1126/science.1196893.

19-Plimpton, S.: Fast Parallel Algorithms for Short-Range Molecular Dynamics. J. Comput. Phys. 117, 1-19 (1995). doi:10.1006/jcph.1995.1039.

20-Humphrey, W., Dalke, A., Schulten, K.: VMD: Visual molecular dynamics. J. Mol. Graph. 14, 33-38 (1996). doi:10.1016/02637855(96)00018-5.

21-Liu, X., Metcalf, T.H., Robinson, J.T., Houston, B.H., Scarpa, F.: Shear Modulus of Monolayer Graphene Prepared by Chemical Vapor Deposition. Nano Lett. 12, 1013-1017 (2012). doi:10.1021/nl204196v.

22-Stuart, S.J., Tutein, A.B., Harrison, J.A.: A reactive potential for hydrocarbons with intermolecular interactions. J. Chem. Phys. 112, 6472-6486 (2000). doi:10.1063/1.481208

23-Liu, F., Ming, P., Li, J.: Ab initio calculation of ideal strength and phonon instability of graphene under tension. Phys. Rev. B. 76, 064120 doi:10.1103/PhysRevB.76.064120.

24-Jin, C., Lan, H., Peng, L., Suenaga, K., Iijima, S.: Deriving Carbon Atomic Chains from Graphene. Phys. Rev. Lett. 102, 205501 (2009). doi:10.1103/PhysRevLett.102.205501. 